\title{
ON THE VISCOELASTIC MIXTURES OF SOLIDS
}

\author{
JOSE R. FERNÁNDEZ ${ }^{1}$, ANTONIO MAGAÑA ${ }^{2}$, MARÍA MASID ${ }^{1}$ AND RAMÓN QUINTANILLA $^{2}$ \\ ${ }^{1}$ Departamento de Matemática Aplicada I, Universidade de Vigo, Escola de Enxeñería de \\ Telecomunicación, Campus As Lagoas Marcosende s/n, 36310 Vigo, Spain. \\ 2 Departament de Matemàtiques, Universitat Politècnica de Catalunya, Escola Superior \\ d'Enginyeries Industrial, Aeroespacial i Audiovisual de Terrassa, C. Colom 11, 08222 Terrassa, \\ Barcelona, Spain.
}

\begin{abstract}
In this paper we analyze an homogeneous and isotropic mixture of viscoelastic solids. We propose conditions to guarantee the coercivity of the internal energy and also of the dissipation, first in dimension two and later in dimension three. We obtain an uniqueness result for the solutions when the dissipation is positive and without any hypothesis over the internal energy. When the internal energy and the dissipation are both positive, we prove the existence of solutions as well as their analyticity. Exponential stability and impossibility of localization of the solutions are immediate consequences.
\end{abstract}

Keywords: viscoelastic mixture, positive internal energy, positive dissipation, uniqueness, existence, analyticity.

\section{INTRODUCTION}

The continuum theory of mixtures has deserved a lot of attention in the recent years. The origin of the current formulation of the thermomechanical theories of mixtures comes from the works of Truesdell and Toupin [1], Kelly [2], Eringen and Ingram [3, 4], Green and Naghdi [5, 6], Müller [7], Dunwoody and Müller [8] and Bowen and Wise [9].

For a deep review of the literature about mixtures with historical references, the reader may consult the papers (or books) of Bowen [10], Atkin and Craine [11, 12], Bedford and Drumheller [13], Samohyl [14], Rajagopal and Tao [15] or Eringen [16, 17]. Some other authors have studied the theory of viscoelastic mixtures. Without trying to be exhaustive, let us highlight the works given in the references [18], [19], [20], [21], [22] and [23].

In this paper we consider an homogeneous and isotropic mixture of viscoelastic materials with a centre of symmetry, and we are interested in knowing the conditions that the constitutive coefficients have to satisfy in order to get the internal energy and the dissipation to be positive definite functions.

Another aim of the paper is to show the exponential decay of the solutions. Therefore, we think that it is suitable to recall here several situations where the thermal and other dissipation mechanisms bring the system determined by a mixture to the exponential stability of the solutions. Some of these situations can be found in the references [24], [25], [26] and [27].

On the one hand, the conditions about the coefficients of the system are relevant when analyzing the materials because the problem will be well posed if the internal energy is positive definite,

Date: June 8, 2017

Corresponding author: Antonio Magaña, antonio.magana@upc.edu, tel. +34937 398206. 
and the stability of the system will be guaranteed if the dissipation is also positive definite. The uniqueness of the solutions is also a question related with the positivity of the dissipation. On the other hand, when numerical simulation studies about the solutions are carried out, the scientist should be provided with a family of conditions about the coefficients that guarantee the well-posedness and stability of the problem.

The plan of the paper is as follows. In Section 2 we recall the basic equations of the theory. Sections 3 and 4 are devoted to obtain conditions about the coefficients of the system of equations for mixtures of thermoviscoelastic materials that make the internal energy and the dissipation to be positive definite. After that, in Sections 5, 6 and 7 we study the uniqueness, the existence and the analyticity of the solutions, respectively. Exponential stability and impossibility of localization will be two consequences. Finally, in Section 8 we state the conclusions.

\section{BASIC EQUATIONS}

As we wrote in the Introduction, we focus our attention into isotropic homogeneous materials with centre of symmetry. We consider a mixture of two linear interacting continua $S_{1}$ and $S_{2}$. For the linear theory, the basic geometric tensors are (see equation (58) from [28]):

$$
e_{i j}=\frac{1}{2}\left(u_{i, j}+u_{j, i}\right), \quad g_{i j}=u_{j, i}+w_{i, j}, \quad d_{i}=u_{i}-w_{i} .
$$

In these expressions $u_{i}$ and $w_{i}$ denote the displacement of each constituent and $d_{i}$ is the relative displacement. It is worth noting that the strain tensor $e_{i j}$ is symmetric, but $g_{i j}$ is not. In particular, in the three-dimensional situation both tensors have nine components, but $e_{i j}$ has only six independent components.

It is known that for isotropic homogeneous materials with centre of symmetry the constitutive equations for the partial tensions and the inner body force are given by (see equation (71) from $[28])$ :

$$
\begin{aligned}
& t_{j i}=(\lambda+\nu) e_{r r} \delta_{j i}+2(\mu+\zeta) e_{j i}+(\alpha+\nu) g_{s s} \delta_{j i}+(2 \gamma+\zeta) g_{i j}+(2 \kappa+\zeta) g_{j i}+t_{j i}^{*} \\
& s_{j i}=\nu e_{r r} \delta_{j i}+2 \zeta e_{i j}+\alpha g_{r r} \delta_{j i}+2 \kappa g_{i j}+2 \gamma g_{j i}+s_{j i}^{*}, \\
& p_{i}=\xi d_{i}+p_{i}^{*},
\end{aligned}
$$

where

$$
\begin{aligned}
& t_{j i}^{*}=\left(\lambda^{*}+\nu_{1}^{*}\right) \dot{e}_{r r} \delta_{j i}+2\left(\mu^{*}+\zeta_{1}^{*}\right) \dot{e}_{j i}+\left(\alpha^{*}+\nu^{*}\right) \dot{g}_{s s} \delta_{j i}+\left(2 \gamma^{*}+\zeta^{*}\right) \dot{g}_{i j}+\left(2 \kappa^{*}+\zeta^{*}\right) \dot{g}_{j i}, \\
& s_{j i}^{*}=\nu_{1}^{*} \dot{e}_{r r} \delta_{j i}+2 \zeta_{1}^{*} \dot{e}_{i j}+\alpha^{*} \dot{g}_{r r} \delta_{j i}+2 \kappa^{*} \dot{g}_{i j}+2 \gamma^{*} \dot{g}_{j i}, \\
& p_{i}^{*}=\xi^{*} \dot{d}_{i} .
\end{aligned}
$$

Here $\lambda, \nu, \mu, \zeta, \alpha, \gamma, \kappa, \xi, \lambda^{*}, \nu_{1}^{*}, \mu^{*}, \zeta_{1}^{*}, \alpha^{*}, \nu^{*}, \gamma^{*}, \zeta^{*}, \kappa^{*}$ and $\xi^{*}$ are the constitutive coefficients over which we will determine the necessary conditions to guarantee the positivity of the internal energy and also of the dissipation.

The evolution equations are given by

$$
t_{j i, j}-p_{i}=\rho_{1} \ddot{u}_{i}, \quad s_{j i, j}+p_{i}=\rho_{2} \ddot{w}_{i} .
$$


Substituting the constitutive equations into the evolution equations, we obtain the field equations:

$$
\left\{\begin{array}{l}
(\lambda+2 \nu+\mu+2 \zeta+\alpha+2 \gamma) u_{j, j i}+(\mu+2 \kappa+2 \zeta) u_{i, j j}+(\alpha+\nu+2 \kappa+\zeta) w_{j, j i}+(2 \gamma+\zeta) w_{i, j j} \\
+\left(\lambda^{*}+\nu^{*}+\nu_{1}^{*}+\mu^{*}+\zeta^{*}+\zeta_{1}^{*}+\alpha^{*}+2 \gamma^{*}\right) \dot{u}_{j, j i}+\left(\mu^{*}+2 \kappa^{*}+\zeta^{*}+\zeta_{1}^{*}\right) \dot{u}_{i, j j} \\
+\left(\alpha^{*}+\nu^{*}+2 \kappa^{*}+\zeta^{*}\right) \dot{w}_{j, j i}+\left(2 \gamma^{*}+\zeta^{*}\right) \dot{w}_{i, j j}-\xi\left(u_{i}-w_{i}\right)-\xi^{*}\left(\dot{u}_{i}-\dot{w}_{i}\right)=\rho_{1} \ddot{u}_{i}, \\
(\nu+\zeta+\alpha+2 \kappa) u_{j, j i}+(\zeta+2 \gamma) u_{i, j j}+(\alpha+2 \gamma) w_{j, j i}+2 \kappa w_{i, j j}+\left(\nu_{1}^{*}+\zeta_{1}^{*}+\alpha^{*}+2 \kappa^{*}\right) \dot{u}_{j, j i} \\
+\left(\zeta_{1}^{*}+2 \gamma^{*}\right) \dot{u}_{i, j j}+\left(\alpha^{*}+2 \gamma^{*}\right) \dot{w}_{j, j i}+2 \kappa^{*} \dot{w}_{i, j j}+\xi\left(u_{i}-w_{i}\right)+\xi^{*}\left(\dot{u}_{i}-\dot{w}_{i}\right)=\rho_{2} \ddot{w}_{i} .
\end{array}\right.
$$

To have a well determined problem we need to impose boundary and initial conditions. Let $\Gamma$ be the domain where the equations are defined and $\partial \Gamma$ its boundary, which we assume regular enough to allow the use of the divergence theorem. As boundary conditions we will assume that

$$
u_{i}(\mathbf{x}, t)=0 \text { and } w_{i}(\mathbf{x}, t)=0 \text { for } \mathbf{x} \in \partial \Gamma,
$$

and as initial conditions we assume that

$$
\begin{aligned}
& u_{i}(\mathbf{x}, 0)=u_{i}^{0}(\mathbf{x}), \quad \dot{u}_{i}(\mathbf{x}, 0)=v_{i}^{0}(\mathbf{x}) \\
& w_{i}(\mathbf{x}, 0)=w_{i}^{0}(\mathbf{x}), \quad \dot{w}_{i}(\mathbf{x}, 0)=z_{i}^{0}(\mathbf{x}) .
\end{aligned}
$$

As we want to set up conditions for the coefficients to make the inner energy and the dissipation positive, we need the expressions of both of them. On the one hand, the internal energy is given by

$$
2 U=\left(t_{j i}-t_{j i}^{*}\right) u_{i, j}+\left(s_{j i}-s_{j i}^{*}\right) w_{i, j}+\left(p_{i}-p_{i}^{*}\right) d_{i},
$$

and therefore,

$$
\begin{aligned}
& 2 U=\lambda e_{r r} e_{s s}+2 \mu e_{j i} e_{j i}+2 \nu e_{r r} g_{s s}+2 \zeta g_{i j} e_{i j}+2 \zeta g_{j i} e_{i j}+\alpha g_{r r} g_{s s}+2 \kappa g_{j i} g_{j i}+ \\
& \quad 2 \gamma g_{j i} g_{i j}+\xi d_{i} d_{i} .
\end{aligned}
$$

On the other hand, the dissipation is given by (see (66) from [28])

$$
\begin{array}{r}
D=\lambda^{*} \dot{e}_{r r} \dot{e}_{s s}+2 \mu^{*} \dot{e}_{j i} \dot{e}_{j i}+\left(\nu^{*}+\nu_{1}^{*}\right) \dot{e}_{r r} \dot{g}_{s s}+\left(\zeta^{*}+\zeta_{1}^{*}\right) \dot{g}_{i j} \dot{e}_{i j}+\left(\zeta^{*}+\zeta_{1}^{*}\right) \dot{g}_{j i} \dot{e}_{i j}+ \\
\alpha^{*} \dot{g}_{r r} \dot{g}_{s s}+2 \kappa^{*} \dot{g}_{j i} \dot{g}_{j i}+2 \gamma^{*} \dot{g}_{j i} \dot{g}_{i j}+\xi^{*} \dot{d}_{i} \dot{d}_{i} .
\end{array}
$$

Material stability requires the nonnegativity of the strain internal energy for all the variations of the strain measures.

In sections 3 and 4 we study the conditions for $U$ and $D$ to be positive in dimension 2 and in dimension 3 , respectively, because the conditions obtained for the first case are not immediately generalizable to the second one.

\section{Conditions in the two-Dimensional Case}

In the two-dimensional theory, functions $U$ and $D$ and their time derivatives have ten variables: $e_{11}, e_{12}, e_{22}, g_{11}, g_{12}, g_{21}, g_{22}, d_{1}, d_{2}$ and $d_{3}$.

We want to obtain the conditions of positive-definiteness of $U$ and $D$. In view of the form of these two quadratic forms, it must be $\xi>0$ for $U$ and $\xi^{*}>0$ for $D$. Therefore, we can remove variables $d_{i}$ and study only the part corresponding to the strain and time derivative of the strain tensors.

In consequence, for the internal energy $U$, the condition of positive-definiteness is equivalent to have $\xi>0$ and that the $7 \times 7$ matrix of the inner product, denoted by $M$ and corresponding 
only to variables $e_{i j}$ and $g_{i j}$, be positive definite. By choosing the order $e_{11}, e_{22}, g_{11}, g_{22}, e_{12}$, $g_{12}$ and $g_{21}$ for the variables, matrix $M$ can be written as follows:

$$
M=\left(\begin{array}{ccccccc}
\lambda+2 \mu & \lambda & 2 \zeta+\nu & \nu & 0 & 0 & 0 \\
\lambda & \lambda+2 \mu & \nu & 2 \zeta+\nu & 0 & 0 & 0 \\
2 \zeta+\nu & \nu & \alpha+2 \gamma+2 \kappa & \alpha & 0 & 0 & 0 \\
\nu & 2 \zeta+\nu & \alpha & \alpha+2 \gamma+2 \kappa & 0 & 0 & 0 \\
0 & 0 & 0 & 0 & 4 \mu & 2 \zeta & 2 \zeta \\
0 & 0 & 0 & 0 & 2 \zeta & 2 \kappa & 2 \gamma \\
0 & 0 & 0 & 0 & 2 \zeta & 2 \gamma & 2 \kappa
\end{array}\right),
$$

which has seven different eigenvalues:

$$
\begin{aligned}
& \text { eig }_{1}=2(\kappa-\gamma) \\
& \text { eig }_{2}=\gamma+\kappa+\mu-\sqrt{(\gamma+\kappa-\mu)^{2}+4 \zeta^{2}} \\
& \text { eig }_{3}=\gamma+\kappa+\mu+\sqrt{(\gamma+\kappa-\mu)^{2}+4 \zeta^{2}} \\
& \text { eig }_{4}=\gamma+\kappa+2 \mu-\sqrt{(\gamma+\kappa-2 \mu)^{2}+8 \zeta^{2}} \\
& \text { eig }_{5}=\gamma+\kappa+2 \mu+\sqrt{(\gamma+\kappa-2 \mu)^{2}+8 \zeta^{2}} \\
& \text { eig }_{6}=\alpha+\gamma+\kappa+\lambda+\mu-\sqrt{(\alpha+\gamma+\kappa-\lambda-\mu)^{2}+4(\zeta+\nu)^{2}} \\
& \text { eig }
\end{aligned}
$$

Remark 3.1. For $b>0$ the numbers $a \pm \sqrt{b}$ are both positive if, and only if, $a>0$ and $a^{2}>b$.

Using Remark 3.1, the eigenvalues of $M$ will be positive if, and only if, the following conditions are satisfied:

$$
\begin{gathered}
\kappa>\gamma \text { for } e^{2} g_{1} \text { positive, } \\
\gamma+\kappa+\mu>0 \text { and }(\gamma+\kappa) \mu>\zeta^{2} \text { for } e i g_{2} \text { and } e i g_{3} \text { positive, } \\
\gamma+\kappa+2 \mu>0 \text { and }(\gamma+\kappa) \mu>\zeta^{2} \text { for } e i g_{4} \text { and } e i g_{5} \text { positive, } \\
\alpha+\gamma+\kappa+\lambda+\mu>0 \text { and }(\alpha+\gamma+\kappa)(\lambda+\mu)>(\zeta+\nu)^{2} \text { for } e i g_{6} \text { and } e i g_{7} \text { positive. }
\end{gathered}
$$

Remark 3.2. Given three real numbers $a, b$ and $c$, then $a+b>0$ and $a b>c^{2}$ if, and only if, $a>0$ and $a b>c^{2}$.

Using Remark 3.2, condition (3.2) reduces to $\mu>0$ and $(\gamma+\kappa) \mu>\zeta^{2}$. Thus, condition (3.3) is unnecessary.

Following the same reasoning, condition (3.4) reduces to $\lambda+\mu>0$ and $(\alpha+\gamma+\kappa)(\lambda+\mu)>$ $(\zeta+\nu)^{2}$.

Theorem 3.3. The quadratic form corresponding to the inner energy $U$ is positive definite if, and only if,

$$
\begin{aligned}
& \kappa>\gamma, \\
& \mu>0, \\
& (\gamma+\kappa) \mu>\zeta^{2}, \\
& \lambda+\mu>0, \\
& (\alpha+\gamma+\kappa)(\lambda+\mu)>(\zeta+\nu)^{2}, \\
& \xi>0 .
\end{aligned}
$$


Making an analogous reasoning for the dissipation $D$, we obtain the following $7 \times 7$ matrix (all the coefficients of $M^{*}$ should have a $*$, but to make $M^{*}$ not too much large, we omit the symbol $*$ in writing of the matrix):

$$
M^{*}=\left(\begin{array}{ccccccc}
\lambda+2 \mu & \lambda & \frac{2 \zeta+2 \zeta_{1}+\nu+\nu_{1}}{2} & \frac{\nu+\nu_{1}}{2} & 0 & 0 & 0 \\
\lambda & \lambda+2 \mu & \frac{\nu+\nu_{1}}{2} & \frac{2 \zeta+2 \zeta_{1}+\nu+\nu_{1}}{2} & 0 & 0 & 0 \\
\frac{2 \zeta+2 \zeta_{1}+\nu+\nu_{1}}{2} & \frac{\nu+\nu_{1}}{2} & \alpha+2 \gamma+2 \kappa & \alpha & 0 & 0 & 0 \\
\frac{\nu+\nu_{1}}{2} & \frac{2 \zeta+2 \zeta_{1}+\nu+\nu_{1}}{2} & \alpha & \alpha+2 \gamma+2 \kappa & 0 & 0 & 0 \\
0 & 0 & 0 & 0 & 4 \mu & \zeta+\zeta_{1} & \zeta+\zeta_{1} \\
0 & 0 & 0 & 0 & \zeta+\zeta_{1} & 2 \kappa & 2 \gamma \\
0 & 0 & 0 & 0 & \zeta+\zeta_{1} & 2 \gamma & 2 \kappa
\end{array}\right) .
$$

Matrix $M^{*}$ has also seven different eigenvalues:

$$
\begin{aligned}
& e i g_{1}^{*}=2\left(\kappa^{*}-\gamma^{*}\right) \\
& e i g_{2}^{*}=\gamma^{*}+\kappa^{*}+\mu^{*}-\sqrt{\left(\gamma^{*}+\kappa^{*}-\mu^{*}\right)^{2}+\left(\zeta^{*}+\zeta_{1}^{*}\right)^{2}} \\
& \text { eig }=\gamma^{*}+\kappa^{*}+\mu^{*}+\sqrt{\left(\gamma^{*}+\kappa^{*}-\mu^{*}\right)^{2}+\left(\zeta^{*}+\zeta_{1}^{*}\right)^{2}} \\
& \text { eig }=\gamma^{*}+\kappa^{*}+2 \mu^{*}-\sqrt{\left(\gamma^{*}+\kappa^{*}-2 \mu^{*}\right)^{2}+2\left(\zeta^{*}+\zeta_{1}^{*}\right)^{2}} \\
& \text { eig }=\gamma^{*}+\kappa^{*}+2 \mu^{*}+\sqrt{\left(\gamma^{*}+\kappa^{*}-2 \mu^{*}\right)^{2}+2\left(\zeta^{*}+\zeta_{1}^{*}\right)^{2}}, \\
& \text { eig }=\alpha^{*}+\gamma^{*}+\kappa^{*}+\lambda^{*}+\mu^{*}-\sqrt{\left(\alpha^{*}+\gamma^{*}+\kappa^{*}-\lambda^{*}-\mu^{*}\right)^{2}+\left(\zeta^{*}+\zeta_{1}^{*}+\nu^{*}+\nu_{1}^{*}\right)^{2}} \\
& \text { eig }
\end{aligned}
$$

The eigenvalues of $M^{*}$ will be positive if, and only if, the following conditions are satisfied:

$$
\left\{\begin{array}{l}
\kappa^{*}>\gamma^{*} \\
\gamma^{*}+\kappa^{*}+\mu^{*}>0 \text { and } 4 \mu^{*}\left(\gamma^{*}+\kappa^{*}\right)>\left(\zeta^{*}+\zeta_{1}^{*}\right)^{2} \\
\gamma^{*}+\kappa^{*}+2 \mu^{*}>0 \text { and } 4 \mu^{*}\left(\gamma^{*}+\kappa^{*}\right)>\left(\zeta^{*}+\zeta_{1}^{*}\right)^{2} \\
\alpha^{*}+\gamma^{*}+\kappa^{*}+\lambda^{*}+\mu^{*}>0 \text { and } 4\left(\lambda^{*}+\mu^{*}\right)\left(\alpha^{*}+\gamma^{*}+\kappa^{*}\right)>\left(\zeta^{*}+\zeta_{1}^{*}+\nu^{*}+\nu_{1}^{*}\right)^{2}
\end{array}\right.
$$

As before, applying Remarks 3.1 and 3.2 these conditions can be summarized in the following result.

Theorem 3.4. The quadratic form corresponding to the dissipation $D$ will be positive definite if, and only if,

$$
\begin{aligned}
& \kappa^{*}>\gamma^{*}, \\
& \mu^{*}>0 \\
& 4 \mu^{*}\left(\gamma^{*}+\kappa^{*}\right)>\left(\zeta^{*}+\zeta_{1}^{*}\right)^{2}, \\
& \lambda^{*}+\mu^{*}>0 \\
& 4\left(\lambda^{*}+\mu^{*}\right)\left(\alpha^{*}+\gamma^{*}+\kappa^{*}\right)>\left(\zeta^{*}+\zeta_{1}^{*}+\nu^{*}+\nu_{1}^{*}\right)^{2}, \\
& \xi^{*}>0 .
\end{aligned}
$$

\section{Conditions in the three-Dimensional CASE}

For the three-dimensional case, $U$ and $D$ have eighteen variables. We impose again that $\xi>0$ and $\xi^{*}>0$ and, as before, we remove the variables of the relative displacement $d_{i}$. Therefore, only variables $e_{i j}$ and $g_{i j}$ remain. Nevertheless, the matrices for $U$ and for $D$ still have fifteen rows and fifteen columns. 
For the internal energy $U$, choosing the variables in the order $e_{11}, e_{22}, e_{33}, g_{11}, g_{22}, g_{33}, e_{12}, e_{13}$, $e_{23}, g_{12}, g_{13}, g_{21}, g_{23}, g_{31}$ and $g_{32}$, matrix $M$ (we abuse a little bit the notation and we denote this matrix as in the two-dimensional case) can be written as follows:

$$
M=\left(\begin{array}{cc}
A & \mathbf{0} \\
\mathbf{0} & B
\end{array}\right)
$$

where

$$
A=\left(\begin{array}{cccccc}
\lambda+2 \mu & \lambda & \lambda & 2 \zeta+\nu & \nu & \nu \\
\lambda & \lambda+2 \mu & \lambda & \nu & 2 \zeta+\nu & \nu \\
\lambda & \lambda & \lambda+2 \mu & \nu & \nu & 2 \zeta+\nu \\
2 \zeta+\nu & \nu & \nu & \alpha+2 \gamma+2 \kappa & \alpha & \alpha \\
\nu & 2 \zeta+\nu & \nu & \alpha & \alpha+2 \gamma+2 \kappa & \alpha \\
\nu & \nu & 2 \zeta+\nu & \alpha & \alpha & \alpha+2 \gamma+2 \kappa
\end{array}\right)
$$

and

$$
B=\left(\begin{array}{ccccccccc}
4 \mu & 0 & 0 & 2 \zeta & 0 & 2 \zeta & 0 & 0 & 0 \\
0 & 4 \mu & 0 & 0 & 2 \zeta & 0 & 0 & 2 \zeta & 0 \\
0 & 0 & 4 \mu & 0 & 0 & 0 & 2 \zeta & 0 & 2 \zeta \\
2 \zeta & 0 & 0 & 2 \kappa & 0 & 2 \gamma & 0 & 0 & 0 \\
0 & 2 \zeta & 0 & 0 & 2 \kappa & 0 & 0 & 2 \gamma & 0 \\
2 \zeta & 0 & 0 & 2 \gamma & 0 & 2 \kappa & 0 & 0 & 0 \\
0 & 0 & 2 \zeta & 0 & 0 & 0 & 2 \kappa & 0 & 2 \gamma \\
0 & 2 \zeta & 0 & 0 & 2 \gamma & 0 & 0 & 2 \kappa & 0 \\
0 & 0 & 2 \zeta & 0 & 0 & 0 & 2 \gamma & 0 & 2 \kappa
\end{array}\right)
$$

As in the two-dimensional case, $M$ has seven different eigenvalues:

$$
\begin{aligned}
& \text { eig }_{1}=2(\kappa-\gamma) \\
& \text { eig }_{2}=\gamma+\kappa+\mu-\sqrt{(\gamma+\kappa-\mu)^{2}+4 \zeta^{2}}, \\
& \text { eig }_{3}=\gamma+\kappa+\mu+\sqrt{(\gamma+\kappa-\mu)^{2}+4 \zeta^{2}}, \\
& \text { eig }_{4}=\gamma+\kappa+2 \mu-\sqrt{(\gamma+\kappa-2 \mu)^{2}+8 \zeta^{2}} \\
& \text { eig }_{5}=\gamma+\kappa+2 \mu+\sqrt{(\gamma+\kappa-2 \mu)^{2}+8 \zeta^{2}} \\
& \text { eig }=\frac{1}{2}\left(3 \alpha+2 \gamma+2 \kappa+3 \lambda+2 \mu-\sqrt{(3 \alpha+2 \gamma+2 \kappa-3 \lambda-2 \mu)^{2}+4(2 \zeta+3 \nu)^{2}}\right), \\
& \text { eig }=\frac{1}{2}\left(3 \alpha+2 \gamma+2 \kappa+3 \lambda+2 \mu+\sqrt{(3 \alpha+2 \gamma+2 \kappa-3 \lambda-2 \mu)^{2}+4(2 \zeta+3 \nu)^{2}}\right) .
\end{aligned}
$$

The eigenvalues of $M$ will be positive if, and only if, the following conditions are satisfied:

$$
\left\{\begin{array}{l}
\kappa>\gamma \\
\gamma+\kappa+\mu>0 \text { and }(\gamma+\kappa) \mu>\zeta^{2}, \\
\gamma+\kappa+2 \mu>0 \text { and }(\gamma+\kappa) \mu>\zeta^{2}, \\
3 \alpha+2 \gamma+2 \kappa+3 \lambda+2 \mu>0 \text { and }(3 \lambda+2 \mu)(3 \alpha+2 \gamma+2 \kappa)>(2 \zeta+3 \nu)^{2} .
\end{array}\right.
$$

Theorem 4.1. The quadratic form corresponding to the internal energy $U$ will be positive definite if, and only if,

$$
\begin{aligned}
& \kappa>\gamma \\
& \mu>0 \\
& (\gamma+\kappa) \mu>\zeta^{2} \\
& 3 \lambda+2 \mu>0 \\
& (3 \lambda+2 \mu)(3 \alpha+2 \gamma+2 \kappa)>(2 \zeta+3 \nu)^{2} \\
& \xi>0
\end{aligned}
$$


A similar analysis can be made for the dissipation $D$. In this case,

$$
M^{*}=\left(\begin{array}{cc}
A^{*} & \mathbf{0} \\
\mathbf{0} & B^{*}
\end{array}\right)
$$

where

$$
A^{*}=\left(\begin{array}{cccccc}
\lambda+2 \mu & \lambda & \lambda & \frac{2 \zeta+2 \zeta_{1}+\nu+\nu_{1}}{2} & \frac{\nu+\nu_{1}}{2} & \frac{\nu+\nu_{1}}{2} \\
\lambda & \lambda+2 \mu & \lambda & \frac{\nu+\nu_{1}}{2} & \frac{2 \zeta+2 \zeta_{1}+\nu+\nu_{1}}{2} & \frac{\nu+\nu_{1}}{2} \\
\lambda & \lambda & \lambda+2 \mu & \frac{\nu+\nu_{1}}{2} & \frac{\nu+\nu_{1}}{2} & \frac{2 \zeta+2 \zeta_{1}+\nu+\nu_{1}}{2} \\
\frac{2 \zeta+2 \zeta_{1}+\nu+\nu_{1}}{2} & \frac{\nu+\nu_{1}}{2} & \frac{\nu+\nu_{1}}{2} & \alpha+2 \gamma+2 \kappa & \alpha & \alpha \\
\frac{\nu+\nu_{1}}{2} & \frac{2 \zeta+2 \zeta_{1}+\nu+\nu_{1}}{2} & \frac{\nu+\nu_{1}}{2} & \alpha & \alpha+2 \gamma+2 \kappa & \alpha \\
\frac{\nu+\nu_{1}}{2} & \frac{\nu+\nu_{1}}{2} & \frac{2 \zeta+2 \zeta_{1}+\nu+\nu_{1}}{2} & \alpha & \alpha & \alpha+2 \gamma+2 \kappa
\end{array}\right)
$$

and

$$
B^{*}=\left(\begin{array}{ccccccccc}
4 \mu & 0 & 0 & \zeta+\zeta_{1} & 0 & \zeta+\zeta_{1} & 0 & 0 & 0 \\
0 & 4 \mu & 0 & 0 & \zeta+\zeta_{1} & 0 & 0 & \zeta+\zeta_{1} & 0 \\
0 & 0 & 4 \mu & 0 & 0 & 0 & \zeta+\zeta_{1} & 0 & \zeta+\zeta_{1} \\
\zeta+\zeta_{1} & 0 & 0 & 2 \kappa & 0 & 2 \gamma & 0 & 0 & 0 \\
0 & \zeta+\zeta_{1} & 0 & 0 & 2 \kappa & 0 & 0 & 2 \gamma & 0 \\
\zeta+\zeta_{1} & 0 & 0 & 2 \gamma & 0 & 2 \kappa & 0 & 0 & 0 \\
0 & 0 & \zeta+\zeta_{1} & 0 & 0 & 0 & 2 \kappa & 0 & 2 \gamma \\
0 & \zeta+\zeta_{1} & 0 & 0 & 2 \gamma & 0 & 0 & 2 \kappa & 0 \\
0 & 0 & \zeta+\zeta_{1} & 0 & 0 & 0 & 2 \gamma & 0 & 2 \kappa
\end{array}\right)
$$

All the coefficients in $A^{*}$ and in $B^{*}$ should have a $*$, but again we prefer to omit it in the matrices to ease the notation and not to enlarge them too much.

Matrix $M^{*}$ has also seven different eigenvalues:

$$
\begin{aligned}
& e i g_{1}^{*}=2\left(\kappa^{*}-\gamma^{*}\right) \\
& e i g_{2}^{*}=\gamma^{*}+\kappa^{*}+\mu^{*}-\sqrt{\left(\gamma^{*}+\kappa^{*}-\mu^{*}\right)^{2}+\left(\zeta^{*}+\zeta_{1}^{*}\right)^{2}}, \\
& e i g_{3}^{*}=\gamma^{*}+\kappa^{*}+\mu^{*}+\sqrt{\left(\gamma^{*}+\kappa^{*}-\mu^{*}\right)^{2}+\left(\zeta^{*}+\zeta_{1}^{*}\right)^{2}} \text {, } \\
& e i g_{4}^{*}=\gamma^{*}+\kappa^{*}+2 \mu^{*}-\sqrt{\left(\gamma^{*}+\kappa^{*}-2 \mu^{*}\right)^{2}+2\left(\zeta^{*}+\zeta_{1}^{*}\right)^{2}}, \\
& e i g_{5}^{*}=\gamma^{*}+\kappa^{*}+2 \mu^{*}+\sqrt{\left(\gamma^{*}+\kappa^{*}-2 \mu^{*}\right)^{2}+2\left(\zeta^{*}+\zeta_{1}^{*}\right)^{2}}, \\
& e i g_{6}^{*}=\frac{1}{2}\left(3 \alpha^{*}+2 \gamma^{*}+2 \kappa^{*}+3 \lambda^{*}+2 \mu^{*}-\sqrt{\left(3 \alpha^{*}+2 \gamma^{*}+2 \kappa^{*}-3 \lambda^{*}-2 \mu^{*}\right)^{2}+\left(2 \zeta^{*}+2 \zeta_{1}^{*}+3 \nu^{*}+3 \nu_{1}^{*}\right)^{2}}\right), \\
& e i g_{7}^{*}=\frac{1}{2}\left(3 \alpha^{*}+2 \gamma^{*}+2 \kappa^{*}+3 \lambda^{*}+2 \mu^{*}+\sqrt{\left(3 \alpha^{*}+2 \gamma^{*}+2 \kappa^{*}-3 \lambda^{*}-2 \mu^{*}\right)^{2}+\left(2 \zeta^{*}+2 \zeta_{1}^{*}+3 \nu^{*}+3 \nu_{1}^{*}\right)^{2}}\right) .
\end{aligned}
$$

The eigenvalues of $M^{*}$ will be positive if, and only if, the following conditions are satisfied:

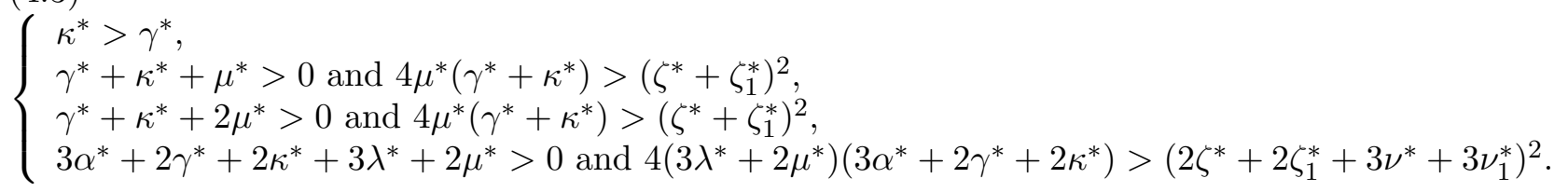


Theorem 4.2. The quadratic form corresponding to the dissipation $D$ will be positive definite if, and only if,

$$
\begin{aligned}
& \kappa^{*}>\gamma^{*}, \\
& \mu^{*}>0 \\
& 4 \mu^{*}\left(\gamma^{*}+\kappa^{*}\right)>\left(\zeta^{*}+\zeta_{1}^{*}\right)^{2}, \\
& 3 \lambda^{*}+2 \mu^{*}>0 \\
& 4\left(3 \lambda^{*}+2 \mu^{*}\right)\left(3 \alpha^{*}+2 \gamma^{*}+2 \kappa^{*}\right)>\left(2 \zeta^{*}+2 \zeta_{1}^{*}+3 \nu^{*}+3 \nu_{1}^{*}\right)^{2}, \\
& \xi^{*}>0 .
\end{aligned}
$$

\section{Uniqueness of SOlutions if $D$ is POSITIVe}

In this section we will see the uniqueness of the solutions supposing only that the dissipation is positive definite (nothing is said about the internal energy). In fact, we will prove that the only solution with null initial conditions and null boundary conditions is the null solution. This fact will prove the uniqueness. We assume in this section that $\rho_{1}$ and $\rho_{2}$ are positive and that $D$ is positive definite.

The conservation of the energy for the system (2.3) with the homogeneous Dirichlet boundary conditions gives

$$
E(t)=\int_{\Gamma}\left(\rho_{1} \dot{u}_{i} \dot{u}_{i}+\rho_{2} \dot{w}_{i} \dot{w}_{i}+2 U\right) d V+2 \int_{0}^{t} \int_{\Gamma} D d V d s=E(0)=0 .
$$

The last equality comes from the assumptions that the initial conditions vanish.

From the above expression, we introduce the following notation:

$$
\begin{aligned}
& I_{1}=\int_{\Gamma}\left(\rho_{1} \dot{u}_{i} \dot{u}_{i}+\rho_{2} \dot{w}_{i} \dot{w}_{i}\right) d V, \\
& I_{2}=\int_{\Gamma} 2 U d V, \\
& I_{3}=2 \int_{0}^{t} \int_{\Gamma} D d V d s,
\end{aligned}
$$

and we define the function $J(t)=I_{1}+I_{3}$. Obviously, $J(t)=-I_{2}$. Therefore,

$$
J(t) \leq C\left(\int_{0}^{t} \int_{\Gamma} e_{i j} e_{i j}+g_{i j} g_{i j}+d_{i} d_{i}\right)^{\frac{1}{2}} I_{3}^{\frac{1}{2}},
$$

where $C$ is a calculable positive constant depending on the constitutive coefficients and the first eigenvalue of the fixed membrane problem (see [29]),

$$
\Delta \Phi+\lambda \Phi=0 \text { on } \Gamma \text { and } \Phi=0 \text { on } \partial \Gamma .
$$

The next step in our analysis uses the Poincaré-type inequality that says that the following estimate

$$
\int_{0}^{t} f^{2}(s) d s \leq \frac{4 t^{2}}{\pi^{2}} \int_{0}^{t}(\dot{f}(s))^{2} d s
$$

holds for every function $f(s)$ such that $f(0)=0$.

Therefore, there exists a positive constant $C_{1}$ such that

$$
\int_{0}^{t} \int_{\Gamma}\left(e_{i j} e_{i j}+g_{i j} g_{i j}+d_{i} d_{i}\right) d V d s \leq t^{2} C_{1} \int_{0}^{t} \int_{\Gamma}\left(\dot{e}_{i j} \dot{e}_{i j}+\dot{g}_{i j} \dot{g}_{i j}+\dot{d}_{i} \dot{d}_{i}\right) d V d s .
$$

Using the positivity of the dissipation function, it can be seen that

$$
J(t) \leq C^{*} t I_{3} \leq C^{*} t J(t)
$$


where $C^{*}$ can be calculated again in terms of the constitutive coefficients and the first eigenvalue of the fixed membrane problem. From (5.2), it follows that $\left(1-C^{*} t\right) J(t) \leq 0$. If we consider $t_{0}=\left(2 C^{*}\right)^{-1}$, then we find that $J(t)$ vanishes in the interval $\left[0, t_{0}\right]$. From the definition of $J(t)$, it follows that $\dot{u}_{i}=0$ and $\dot{w}_{i}=0$ for every $t \leq t_{0}$. Thus, we have proved that the problem has only the null solution in the interval $\left[0, t_{0}\right]$. Applying the same argument to the problem determined by the field equations, the same boundary conditions and the null initial data at the initial instant $t_{0}$, that is, $\dot{u}_{i}\left(\boldsymbol{x}, t_{0}\right)=0, \dot{w}_{i}\left(\boldsymbol{x}, t_{0}\right)=0$, we conclude that $u_{i}=0$ and $w_{i}=0$ for every $t \leq 2 t_{0}$. The following theorem is proved applying recurrently this argument.

Theorem 5.1. Let us assume that $\rho_{1}>0, \rho_{2}>0$ and that $D$ is positive definite. Then the Dirichlet initial boundary value problem has at most one solution.

\section{Existence of SOLUTiON}

In this section we use the results of the semigroup of linear operators theory to obtain an existence theorem. Though other boundary conditions could be proposed, we restrict our attention to the boundary conditions proposed in (2.4).

In the remaining part of the paper we assume that $D$ is positive definite and that the internal energy density $U$ is a positive definite quadratic form (that means that all the inequalities for the coefficients that we have seen before are satisfied). We assume again that $\rho_{1}$ and $\rho_{2}$ are positive.

We now transform the boundary-initial value problem defined by system (2.3), boundary conditions (2.4) and initial conditions (2.5) into an abstract problem on a suitable Hilbert space. We denote

$$
Z=\left\{\mathbf{U}=(\mathbf{u}, \mathbf{v}, \mathbf{w}, \mathbf{z}): u_{i}, w_{i} \in W_{0}^{1,2}(\Gamma), v_{i}, z_{i} \in L^{2}(\Gamma)\right\},
$$

where $W_{0}^{1,2}(\Gamma)$ and $L^{2}(\Gamma)$ are the usual Sobolev spaces, which take values in the complex field.

Let us consider the following operators:

$$
\begin{aligned}
& A_{i}(\mathbf{u})=\frac{1}{\rho_{1}}\left[(\mu+2 \kappa+2 \zeta) u_{i, j j}+(\lambda+2 \nu+\mu+2 \zeta+\alpha+2 \gamma) u_{j, j i}-\xi u_{i}\right], \\
& A_{i}^{*}(\mathbf{v})=\frac{1}{\rho_{1}}\left[\left(\mu^{*}+2 \kappa^{*}+\zeta^{*}+\zeta_{1}^{*}\right) v_{i, j j}+\left(\lambda^{*}+\nu^{*}+\nu_{1}^{*}+\mu^{*}+\zeta^{*}+\zeta_{1}^{*}+\alpha^{*}+2 \gamma^{*}\right) v_{j, j i}-\xi^{*} v_{i}\right], \\
& B_{i}(\mathbf{w})=\frac{1}{\rho_{1}}\left[(2 \gamma+\zeta) w_{i, j j}+(\alpha+\nu+2 \kappa+\zeta) w_{j, j i}+\xi w_{i}\right], \\
& B_{i}^{*}(\mathbf{z})=\frac{1}{\rho_{1}}\left[\left(2 \gamma^{*}+\zeta^{*}\right) z_{i, j j}+\left(\alpha^{*}+\nu^{*}+2 \kappa^{*}+\zeta^{*}\right) z_{j, j i}+\xi^{*} z_{i}\right], \\
& C_{i}(\mathbf{u})=\frac{1}{\rho_{2}}\left[(\zeta+2 \gamma) u_{i, j j}+(\nu+\zeta+\alpha+2 \kappa) u_{j, j i}+\xi u_{i}\right], \\
& C_{i}^{*}(\mathbf{v})=\frac{1}{\rho_{2}}\left[\left(\zeta_{1}^{*}+2 \gamma^{*}\right) v_{i, j j}+\left(\nu_{1}^{*}+\zeta_{1}^{*}+\alpha^{*}+2 \kappa^{*}\right) v_{j, j i}+\xi^{*} v_{i}\right], \\
& D_{i}(\mathbf{w})=\frac{1}{\rho_{2}}\left[2 \kappa w_{i, j j}+(\alpha+2 \gamma) w_{j, j i}-\xi w_{i}\right], \\
& D_{i}^{*}(\mathbf{z})=\frac{1}{\rho_{2}}\left[2 \kappa^{*} z_{i, j j}+\left(\alpha^{*}+2 \gamma^{*}\right) z_{j, j i}-\xi^{*} z_{i}\right] .
\end{aligned}
$$

We denote

$$
\mathcal{D}=\left(\mathbf{W}_{0}^{1,2} \cap \mathbf{W}^{2,2}\right) \times\left(\mathbf{W}_{0}^{1,2} \cap \mathbf{W}^{2,2}\right) \times\left(\mathbf{W}_{0}^{1,2} \cap \mathbf{W}^{2,2}\right) \times\left(\mathbf{W}_{0}^{1,2} \cap \mathbf{W}^{2,2}\right) .
$$

Let $\mathcal{A}$ be the matrix operator defined on $\mathcal{D}$ by

$$
\mathcal{A}=\left(\begin{array}{cccc}
\mathbf{0} & \mathbf{I d} & \mathbf{0} & \mathbf{0} \\
\mathbf{A} & \mathbf{A}^{*} & \mathbf{B} & \mathbf{B}^{*} \\
\mathbf{0} & \mathbf{0} & \mathbf{0} & \mathbf{I d} \\
\mathbf{C} & \mathbf{C}^{*} & \mathbf{D} & \mathbf{D}^{*}
\end{array}\right)
$$


where $\mathbf{A}=\left(A_{i}\right), \mathbf{A}^{*}=\left(A_{i}^{*}\right), \mathbf{B}=\left(B_{i}\right), \mathbf{B}^{*}=\left(B_{i}^{*}\right), \mathbf{C}=\left(C_{i}\right), \mathbf{C}^{*}=\left(C_{i}^{*}\right), \mathbf{D}=\left(D_{i}\right), \mathbf{D}^{*}=\left(D_{i}^{*}\right)$ and $\mathbf{I d}$ represents the identity in the respective space. We note that the domain of $\mathcal{A}$ contains $\mathcal{D}$ which is dense in $\mathcal{Z}$.

The initial boundary value problem $(2.3),(2.4),(2.5)$ can be transformed into the following abstract equation in the Hilbert space $\mathcal{Z}$,

$$
\frac{d \mathbf{U}}{d t}=\mathcal{A} \mathbf{U}(t), \quad \mathbf{U}(0)=\mathbf{U}_{0},
$$

where

$$
\mathbf{U}_{0}=\left(u_{i}^{0}, v_{i}^{0}, w_{i}^{0}, z_{i}^{0}\right) .
$$

We introduce in $\mathcal{Z}$ the inner product

$$
<\mathbf{U}, \mathbf{U}^{\star}>=\frac{1}{2} \int_{\Gamma}\left(\rho_{1} v_{i}{\overline{v_{i}}}^{\star}+\rho_{2} z_{i}{\overline{z_{i}}}^{\star}+\mathcal{M}\left[\mathbf{U}^{\mathbf{0}}, \mathbf{U}^{\mathbf{0}^{\star}}\right]\right) d V
$$

where

$$
\begin{gathered}
\mathbf{U}=(\mathbf{u}, \mathbf{v}, \mathbf{w}, \mathbf{z}), \mathbf{U}^{\star}=\left(\mathbf{u}^{\star}, \mathbf{v}^{\star}, \mathbf{w}^{\star}, \mathbf{z}^{\star}\right), \\
\mathbf{U}^{0}=(\mathbf{u}, \mathbf{w}), \mathbf{U}^{\mathbf{0}^{\star}}=\left(\mathbf{u}^{\star}, \mathbf{w}^{\star}\right)
\end{gathered}
$$

and

$$
\begin{array}{r}
\mathcal{M}\left[\mathbf{U}^{0}, \mathbf{U}^{0^{\star}}\right]=\lambda e_{r r} \overline{e_{s s}^{\star}}+2 \mu e_{j i} \overline{e_{j i}^{\star}}+\nu\left(e_{r r} \overline{g_{s s}^{\star}}+g_{s s} \overline{e_{r r}^{\star}}\right)+\zeta\left(e_{i j} \overline{g_{i j}^{\star}}+g_{i j} \overline{e_{i j}^{\star}}\right)+ \\
\zeta\left(e_{i j} \overline{g_{j i}^{\star}}+g_{j i} \overline{e_{i j}^{\star}}\right)+\alpha g_{r r} \overline{g_{s s}^{\star}}+2 \kappa g_{j i} \overline{g_{j i}^{\star}}+2 \gamma g_{i j} \overline{g_{j i}^{\star}}+\xi d_{i} \overline{d_{i}^{\star}} .
\end{array}
$$

Here, as usual a bar over a variable denotes its complex conjugate.

We note that (6.5) defines a norm which is given by

$$
\begin{gathered}
\|(\mathbf{u}, \mathbf{v}, \mathbf{w}, \mathbf{z})\|^{2}=\frac{1}{2} \int_{\Gamma}\left(\rho_{1} v_{i} \overline{v_{i}}+\rho_{2} z_{i} \overline{z_{i}}+\lambda e_{r r} \overline{e_{s s}}+2 \mu e_{j i} \overline{e_{j i}}+\nu\left(e_{r r} \overline{g_{s s}}+g_{s s} \overline{e_{r r}}\right)+\right. \\
\left.\zeta\left(e_{i j} \overline{g_{i j}}+g_{i j} \overline{e_{i j}}\right)+\zeta\left(e_{i j} \overline{g_{j i}}+g_{j i} \overline{e_{i j}}\right)+\alpha g_{r r} \overline{g_{s s}}+2 \kappa g_{j i} \overline{g_{j i}}+2 \gamma g_{i j} \overline{g_{j i}}+\xi d_{i} \overline{d_{i}}\right) d V .
\end{gathered}
$$

This norm is equivalent to the usual norm in $\mathcal{Z}$ whenever the coefficients satisfy (3.5) or (4.2) when working in dimension 2 or 3 , respectively.

Lemma 6.1. The operator $\mathcal{A}$ has the property

$$
\Re<\mathcal{A} \mathbf{U}, \mathbf{U}>\leq 0,
$$

for any $\mathbf{U} \in \mathcal{D}$, where the inner product $<., .>$ is defined in (6.5).

Proof. Let $\mathbf{U}=(\mathbf{u}, \mathbf{v}, \mathbf{w}, \mathbf{z}) \in \mathcal{D}$. We denote $f_{i j}=\frac{1}{2}\left(v_{i, j}+v_{j, i}\right), h_{i j}=v_{j, i}+z_{i, j}$ and $l_{i}=v_{i}-z_{i}$.

Using the divergence theorem and the boundary conditions we have

$$
\begin{aligned}
& <\mathcal{A} \mathbf{U}, \mathbf{U}>=-\frac{1}{2} \int_{\Gamma}\left(\lambda^{*} f_{r r} \bar{f}_{s s}+2 \mu^{*} f_{i j} \bar{f}_{i j}+\frac{1}{2}\left(\nu^{*}+\nu_{1}^{*}\right)\left(f_{r r} \bar{h}_{s s}+\bar{f}_{r r} h_{s s}\right)+\right. \\
& \left.\frac{1}{2}\left(\zeta^{*}+\zeta_{1}^{*}\right)\left(f_{i j} \bar{h}_{j i}+\bar{f}_{i j} h_{j i}\right)+\alpha^{*} h_{r r} \bar{h}_{s s}+2 \kappa^{*} h_{i j} \bar{h}_{j i}+2 \gamma^{*} h_{i j} \bar{h}_{i j}+\xi^{*} l_{i} \bar{l}_{i}\right) d V .
\end{aligned}
$$

Therefore, if the coefficients satisfy (3.7) or (4.4), depending on the dimension of $\Gamma$, then it will be $\Re<\mathcal{A} \mathbf{U}, \mathbf{U}>\leq 0$.

Lemma 6.2. The operator $\mathcal{A}$ satisfies the condition $0 \in \varrho(\mathcal{A})$. 
Proof. Let $\mathbf{U}^{*}=\left(\mathbf{u}^{*}, \mathbf{v}^{*}, \mathbf{w}^{*}, \mathbf{z}^{*}\right) \in \mathcal{Z}$. We must show that the equation

$$
\mathcal{A} \mathbf{U}=\mathbf{U}^{*}
$$

has a solution $\mathbf{U}=(\mathbf{u}, \mathbf{v}, \mathbf{w}, \mathbf{z}) \in \mathcal{D}$. If we write the matrix and we operate, we find the following system:

$$
\begin{aligned}
& \mathbf{v}=\mathbf{u}^{*} \\
& \mathbf{A} \mathbf{u}+\mathbf{A}^{*} \mathbf{v}+\mathbf{B w}+\mathbf{B}^{*} \mathbf{z}=\mathbf{v}^{*} \\
& \mathbf{z}=\mathbf{w}^{*} \\
& \mathbf{C u}+\mathbf{C}^{*} \mathbf{v}+\mathbf{D w}+\mathbf{D}^{*} \mathbf{z}=\mathbf{z}^{*}
\end{aligned}
$$

Substituting the first and the third equations into the others, we obtain the following system with unknowns $\mathbf{u}$ and $\mathbf{w}$ :

$$
\begin{aligned}
& \mathbf{A u}+\mathbf{B w}=\mathbf{v}^{*}-\mathbf{A}^{*} \mathbf{u}^{*}-\mathbf{B}^{*} \mathbf{w}^{*} \\
& \mathbf{C u}+\mathbf{D} \mathbf{w}=\mathbf{z}^{*}-\mathbf{C}^{*} \mathbf{u}^{*}-\mathbf{D}^{*} \mathbf{w}^{*}
\end{aligned}
$$

Notice that

$$
\left(\mathbf{v}^{*}-\mathbf{A}^{*} \mathbf{u}^{*}-\mathbf{B}^{*} \mathbf{w}^{*}, \mathbf{z}^{*}-\mathbf{C}^{*} \mathbf{u}^{*}-\mathbf{D}^{*} \mathbf{w}^{*}\right) \in \mathbf{W}^{-1,2} \times \mathbf{W}^{-1,2} .
$$

On the other side,

$$
\mathcal{B}\left((\mathbf{u}, \mathbf{w}),\left(\mathbf{u}^{*}, \mathbf{w}^{*}\right)\right)=\left\langle(\mathbf{A u}+\mathbf{B w}, \mathbf{C u}+\mathbf{D w}),\left(\rho_{1} \mathbf{u}^{*}, \rho_{2} \mathbf{w}^{*}\right)\right\rangle
$$

defines a coercive and bounded bilinear form on $\mathbf{W}_{0}^{1,2} \times \mathbf{W}_{0}^{1,2}$. Hence, in $\mathbf{W}_{0}^{1,2} \times \mathbf{W}_{0}^{1,2}$ the LaxMilgram theorem implies the existence of a solution to the system of equations (6.10). Thus, equation (6.8) has also a solution.

Theorem 6.3. The operator $\mathcal{A}$ generates a semigroup of contractions in $\mathcal{Z}$.

The proof follows from the above lemmas and the Lumer-Phillips corollary to the Hille-Yosida theorem.

Finally, as a consequence, we have the following result.

Theorem 6.4. Assume that $\mathbf{U}_{0} \in \mathcal{D}$. Then, there exists a unique solution $\mathbf{U}(t) \in$ $C^{1}([0, \infty), \mathcal{Z}) \cap C^{0}([0, \infty), \mathcal{D})$ to problem $(6.3)$.

\section{Analyticity of SOlutions}

In this section we prove the analyticity of the solutions to problem (6.3).

In order to prove the main result of this section we will use a theorem that can be found in the classical book of Liu and Zheng [30].

Theorem 7.1. Let us consider $S(t)=e^{\mathcal{A} t}$ a $C_{0}$-semigroup of contractions generated by the operator $\mathcal{A}$ in the Hilbert space $\mathcal{Z}$. Suppose that $\varrho(\mathcal{A}) \supseteq\{i \beta ; \beta \in \mathbb{R}\} \equiv i \mathbb{R}$. Then $S(t)$ is analytic if and only if

$$
\varlimsup_{|\beta| \rightarrow \infty}\left\|\beta(i \beta \mathcal{I}-\mathcal{A})^{-1}\right\|<\infty, \quad \beta \in \mathbb{R}
$$

holds. 
To apply this theorem to our situation, we need to consider the resolvent equation

$$
\lambda \mathbf{U}-\mathcal{A} \mathbf{U}=\mathbf{F},
$$

where $\mathbf{U}=(\mathbf{u}, \mathbf{v}, \mathbf{w}, \mathbf{z})$ and $\mathbf{F}=\left(\mathbf{f}_{1}, \mathbf{f}_{2}, \mathbf{f}_{3}, \mathbf{f}_{4}\right)$. We shall take $\lambda=i \delta$, with $\delta \in \mathbb{R}$. Therefore, our equation becomes

$$
\begin{aligned}
& i \delta \mathbf{u}-\mathbf{v}=\mathbf{f}_{1}, \\
& i \delta \mathbf{v}-\mathbf{A u}-\mathbf{A}^{*} \mathbf{v}-\mathbf{B w}-\mathbf{B}^{*} \mathbf{z}=\mathbf{f}_{2}, \\
& i \delta \mathbf{w}-\mathbf{z}=\mathbf{f}_{3}, \\
& i \delta \mathbf{z}-\mathbf{C u}-\mathbf{C}^{*} \mathbf{v}-\mathbf{D w}-\mathbf{D}^{*} \mathbf{z}=\mathbf{f}_{4} .
\end{aligned}
$$

Lemma 7.2. For any $\mathbf{F} \in \mathcal{Z}$ there exists a positive constant $C$ such that

$$
\int_{\Gamma}\left(f_{i j} \bar{f}_{i j}+h_{i j} \bar{h}_{i j}+l_{i} \bar{l}_{i}\right) d V \leq C\|\mathbf{F}\|_{\mathcal{Z}}\|\mathbf{U}\|_{\mathcal{Z}}
$$

where, as before,

$$
f_{i j}=\frac{1}{2}\left(v_{i, j}+v_{j, i}\right), \quad h_{i j}=v_{j, i}+z_{i, j} \text { and } l_{i}=v_{i}-z_{i}
$$

Proof. If we multiply the first equation of (7.1) by $-\mathbf{A} \overline{\mathbf{u}}-\mathbf{B} \overline{\mathbf{w}}$, the second one by $\overline{\mathbf{v}}$, the third one by $-\mathbf{C} \overline{\mathbf{u}}-\mathbf{D} \overline{\mathbf{w}}$, the fourth one by $\overline{\mathbf{z}}$ and we add all the results we obtain at the left-hand side the following expression:

$$
\begin{array}{r}
i \delta(-\langle\mathbf{u}, \mathbf{A} \mathbf{u}+\mathbf{B w}\rangle-\langle\mathbf{w}, \mathbf{C u}+\mathbf{D} \mathbf{w}\rangle+\langle\mathbf{v}, \mathbf{v}\rangle+\langle\mathbf{z}, \mathbf{z}\rangle)+ \\
\langle\mathbf{v}, \mathbf{A u}+\mathbf{B w}\rangle-\langle\mathbf{A u}+\mathbf{B w}, \mathbf{v}\rangle+\langle\mathbf{z}, \mathbf{C u}+\mathbf{D} \mathbf{w}\rangle-\langle\mathbf{C u}+\mathbf{D w}, \mathbf{z}\rangle- \\
\left\langle\mathbf{A}^{*} \mathbf{v}+\mathbf{B}^{*} \mathbf{z}, \mathbf{v}\right\rangle-\left\langle\mathbf{C}^{*} \mathbf{v}+\mathbf{D}^{*} \mathbf{z}, \mathbf{z}\right\rangle .
\end{array}
$$

Moreover, the norm of the right-hand side is bounded by $C_{1}\|\mathbf{F}\|_{\mathcal{Z}}\|\mathbf{U}\|_{\mathcal{Z}}$, for a positive constant $C_{1}$.

Notice that the first line of (7.2) becomes

$$
\begin{array}{r}
i \delta \int_{\Gamma}\left(\rho_{1} v_{i} \overline{v_{i}}+\rho_{2} z_{i} \overline{z_{i}}+\lambda e_{r r} \overline{e_{s s}}+2 \mu e_{j i} \overline{e_{j i}}+\nu\left(e_{r r} \overline{g_{s s}}+g_{s s} \overline{e_{r r}}\right)+\zeta\left(e_{i j} \overline{g_{i j}}+g_{i j} \overline{e_{i j}}\right)+\right. \\
\left.\zeta\left(e_{i j} \overline{g_{j i}}+g_{j i} \overline{e_{i j}}\right)+\alpha g_{r r} \overline{g_{s s}}+2 \kappa g_{j i} \overline{g_{j i}}+2 \gamma g_{i j} \overline{g_{j i}}+\xi d_{i} \overline{d_{i}}\right) d V,
\end{array}
$$

and therefore, it is clear that this number is imaginary.

The second line of (7.2) is also imaginary, as it is obtained from the difference of conjugate complex numbers.

Finally, recalling the definition of the operators $\mathbf{A}^{*}, \mathbf{B}^{*}, \mathbf{C}^{*}$ and $\mathbf{D}^{*}$, from the third line we obtain the dissipation, which is positive.

Lemma 7.3. For any $\mathbf{F} \in \mathcal{Z}$ there exists a positive constant $C$ such that

$$
|\delta|\|\mathbf{U}\|_{\mathcal{Z}} \leq C\|\mathbf{F}\|_{\mathcal{Z}} \quad \forall \delta \in \mathbb{R},
$$

where $\mathbf{U}$ is the solution to problem (7.1).

Proof. Now we multiply the first equation of (7.1) by $-i(\mathbf{A} \overline{\mathbf{u}}+\mathbf{B} \overline{\mathbf{w}})$, the second one by $i \overline{\mathbf{v}}$, the third one by $-i(\mathbf{C} \overline{\mathbf{u}}+\mathbf{D} \overline{\mathbf{w}})$, the fourth one by $i \overline{\mathbf{z}}$ and we add all the results. Notice that if $\langle x, y\rangle=\Re\langle x, y\rangle+i \Im\langle x, y\rangle$, then $\langle x, i y\rangle=\Im\langle x, y\rangle-i \Re\langle x, y\rangle$. That means that now the real part of the sum of the left-hand side is just the imaginary part of (7.2).

The norm of the right-hand side is bounded by $C_{2}\|\mathbf{F}\|_{\mathcal{Z}}\|\mathbf{U}\|_{\mathcal{Z}}$, for a positive constant $C_{2}$. 
Notice that

$$
\begin{array}{r}
-i(\langle\mathbf{v}, \mathbf{A} \mathbf{u}+\mathbf{B w}\rangle-\langle\mathbf{A u}+\mathbf{B w}, \mathbf{v}\rangle+\langle\mathbf{z}, \mathbf{C u}+\mathbf{D w}\rangle-\langle\mathbf{C u}+\mathbf{D w}, \mathbf{z}\rangle)= \\
-2 \Im \mathcal{M}[(\mathbf{v}, \mathbf{z}),(\mathbf{u}, \mathbf{w})],
\end{array}
$$

and, hence, it is real.

Taking into account the first line of (7.2), now we obtain another real part:

$$
\begin{array}{r}
\delta \int_{\Gamma}\left(\rho_{1} v_{i} \overline{v_{i}}+\rho_{2} z_{i} \overline{z_{i}}+\lambda e_{r r} \overline{e_{s s}}+2 \mu e_{j i} \overline{e_{j i}}+\nu\left(e_{r r} \overline{g_{s s}}+g_{s s} \overline{e_{r r}}\right)+\zeta\left(e_{i j} \overline{g_{i j}}+g_{i j} \overline{e_{i j}}\right)+\right. \\
\left.\zeta\left(e_{i j} \overline{g_{j i}}+g_{j i} \overline{e_{i j}}\right)+\alpha g_{r r} \overline{g_{s s}}+2 \kappa g_{j i} \overline{g_{j i}}+2 \gamma g_{i j} \overline{g_{j i}}+\xi d_{i} \overline{d_{i}}\right) d V .
\end{array}
$$

From Lemma 7.2 we know that there exists a positive constant $C_{1}$ such that

$$
2 \Im \mathcal{M}[(\mathbf{v}, \mathbf{z}),(\mathbf{u}, \mathbf{w})] \leq C_{1}\|\mathbf{U}\|^{1 / 2}\|\mathbf{F}\|^{1 / 2}\|\mathbf{U}\|
$$

Therefore, we obtain

$$
\begin{array}{r}
\delta \int_{\Gamma}\left(\rho_{1} v_{i} \overline{v_{i}}+\rho_{2} z_{i} \overline{z_{i}}+\lambda e_{r r} \overline{e_{s s}}+2 \mu e_{j i} \overline{e_{j i}}+\nu\left(e_{r r} \overline{g_{s s}}+g_{s s} \overline{e_{r r}}\right)+\zeta\left(e_{i j} \overline{g_{i j}}+g_{i j} \overline{e_{i j}}\right)+\right. \\
\left.\zeta\left(e_{i j} \overline{g_{j i}}+g_{j i} \overline{e_{i j}}\right)+\alpha g_{r r} \overline{g_{s s}}+2 \kappa g_{j i} \overline{g_{j i}}+2 \gamma g_{i j} \overline{g_{j i}}+\xi d_{i} \overline{d_{i}}\right) d V \leq \\
2 \Im \mathcal{M}[(\mathbf{v}, \mathbf{z}),(\mathbf{u}, \mathbf{w})]+C_{3}\|\mathbf{F}\|\|\mathbf{U}\|,
\end{array}
$$

and, hence,

$$
|\delta|\|\mathbf{U}\|^{2} \leq C_{4}\|\mathbf{U}\|^{3 / 2}\|\mathbf{F}\|^{1 / 2}+C_{5}\|\mathbf{U}\|\|\mathbf{F}\| .
$$

From this inequality we get that

$$
|\delta|\|\mathbf{U}\| \leq C_{6}\|\mathbf{F}\|,
$$

where $C_{6}>0$ and $\delta$ is sufficiently greater.

Theorem 7.4. The semigroup generated by the operator $\mathcal{A}$ is analytic.

Proof. Since $\mathcal{A}$ is the infinitesimal generator of a strongly continuous semigroup, $\mathbb{R}_{+} \in \varrho(\mathcal{A})$ and as $0 \in \varrho(\mathcal{A})$, we have $i \mathbb{R} \subset \varrho(\mathcal{A})$. From Lemma 7.3 we have

$$
\left\|\delta(i \delta \mathcal{I}-\mathcal{A})^{-1} \mathbf{F}\right\|_{\mathcal{Z}}=|\delta|\|\mathbf{U}\|_{\mathcal{Z}} \leq C\|\mathbf{F}\|_{\mathcal{Z}}
$$

Then,

$$
\left.\varlimsup_{|\delta| \rightarrow \infty} \| \delta(i \delta \mathcal{I}-\mathcal{A})^{-1}\right) \|<\infty .
$$

Corollary 7.5. System (2.3) is exponentially stable. That is, there exist two positive constants $M$ and $\omega$ such that $\|\mathbf{U}(t)\| \leq M e^{-\omega t}\|\mathbf{U}(0)\|$.

Another consequence of the analyticity of the solutions is the impossibility of localization. That means that the only solution that can be identically zero after a finite period of time is the null solution.

Corollary 7.6. Let $(\mathbf{u}, \mathbf{w})$ be a solution to system (2.3) with boundary conditions (2.4) and initial conditions (2.5) such that $\mathbf{u}=\mathbf{w} \equiv 0$ after a finite time $t_{0}>0$. Then, $\mathbf{u}=\mathbf{w} \equiv 0$ for every $t \geq 0$. 


\section{Conclusions}

In this paper we have investigated different issues concerning homogeneous and isotropic viscoelastic mixtures. We can summarize the results in the following three items:

(1) Suitable conditions for the constitutive coefficients are given to guarantee the positivity of the internal energy and also the positivity of the dissipation. We have studied separately the two-dimensional and three-dimensional cases.

(2) The uniqueness of the solutions, when the dissipation is positive and without any additional condition on the internal energy, has been proved.

(3) Finally, existence and analyticity of the solutions when the internal energy and the dissipation are both positive have been obtained. Exponential stability and impossibility of localization of the solutions are immediate consequences.

\section{ACKNOWLEDGMENTS}

The work of J.R. Fernández was supported by the Ministerio de Economía and Competitividad under the research project MTM2015-66640-P (with the participation of FEDER).

The work of A. Magaña and R. Quintanilla is supported by projects "Análisis Matemático de las Ecuaciones en Derivadas Parciales de la Termomecánica" (MTM2013-42004-P) and "Análisis Matemático de Problemas de la Termomecánica" (MTM2016-74934-P), (AEI/FEDER, UE) of the Spanish Ministry of Economy and Competitiveness.

We thank professor D. Ieşan for his useful comments and an anonymous referee for helping us to improve the paper.

\section{REFERENCES}

[1] Truesdell, C. and Toupin, R. A. The Classical Field Theories, in (Flügge, S., ed.) Handbuch der Physik, vol. III/3, Springer-Verlag, Berlin, 1960.

[2] Kelly, P. A Reacting Continuum. International Journal of Engineering Science, vol. 2, pp. 129-153, 1964.

[3] Eringen, A. C. and Ingram, J. D. A Continuum Theory of Chemically Reacting Media-I. International Journal of Engineering Science, vol. 3, pp. 197-212, 1965.

[4] Ingram, J. D. and Eringen, A. C. A Continuum Theory of Chemically Reacting Media-II. Constitutive Equations of Reacting Fluids Mixtures. International Journal of Engineering Science, vol. 4, pp. 289-322, 1967.

[5] Green, A. E. and Naghdi, P. M. A Dynamical Theory of Interacting Continua. International Journal of Engineering Science, vol. 3, pp. 231-241, 1965.

[6] Green, A. E. and Naghdi, P. M. A Note on Mixtures. International Journal of Engineering Science, vol. 6, pp. 631-635, 1968.

[7] Müller, I. A Thermodynamic Theory of Mixtures of Fluids. Archive for Rational Mechanics and Analysis, vol. 28, pp. 1-39, 1968.

[8] Dunwoody, N. and Müller, I. A Thermodynamic Theory of Two Chemically Reacting Ideal Gases with Different Temperatures. Archive for Rational Mechanics and Analysis, vol. 29, pp. 344-369, 1968.

[9] Bowen, R. M. and Wiese, J. C.. Diffusion in Mixtures of Elastic Material. International Journal of Engineering Science, vol. 7, pp. 689-722, 1969.

[10] Bowen, R. M. Theory of Mixtures, in (Eringen, A. C., ed.) Continuum Physics, vol. III, pp. 3-120, Academic Press, New York, 1976.

[11] Atkin, R. J. and Craine, R. E. Continuum Theories of Mixtures. Basic Theory and Historial Development. Quarterly Journal of Mechanics and Applied Mathematics, vol. 29, pp. 209-245, 1972.

[12] Atkin, R. J. and Craine, R. E. Continuum Theories of Mixtures. Applications. IMA Journal of Applied Mathematics, vol. 17, pp. 153-207, 1976. 
[13] Bedford, A. and Drumhelles, D. S. Theory of Immiscible and Structured Mixtures. International Journal of Engineering Science, vol. 21, pp. 153-207, 1983.

[14] Samohyl, I. Thermodynamics of Irreversible Processes in Fluid Mixtures. Teubner Verlag, Leipzig, 1987.

[15] Rajagopal, K. R. and Tao, L. Mechanics of Mixtures. World Scientific, Singapore, 1995.

[16] Eringen, A. C. Micropolar Mixture Theory of Porous Media. Journal of Applied Physics, vol. 94, pp. 41844190, 2003

[17] Eringen, A. C. A Mixture Theory for Geophysical Fluids. Nonlinear Processes in Geophysics, vol. 11, pp. 75-82, 2004.

[18] Marinov, P. Toward a Thermoviscoelastic Theory of Two Component Materials. Journal of Applied Physics, vol. 16, pp. 533-555, 1978.

[19] McCarthy, M. F. and Tiersten, H. F. A Theory of Viscoelastic Composites Modeled as Interpretating Solid Continua with Memory. Archive for Rational Mechanics and Analysis, vol. 81, pp. 21-51, 1983.

[20] Hills, R. N. and Roberts, P. H. Relaxation Effects in a Mixed Phase Region. Journal of Non-Equilibrium Thermodynamics, vol. 12, pp. 169-181, 1987.

[21] Hills, R. N. and Roberts, P. H. On the Formulation of Diffusive Mixture Theories for 2-Phase Regions. Journal of Engineering Mathematics, vol. 22, pp. 93-100, 1988.

[22] Aboudi, J. Micromechanical Modelling of Finite Viscoelastic Multiphase Composites. Journal of Applied Mathematics and Physics (ZAMP), vol. 51, pp. 114-134, 2000.

[23] Ieşan, D. and Quintanilla, R. On a Theory of Interacting Continua with Memory. Journal of Thermal Stresses, vol. 25, pp. 1161-1178, 2002.

[24] Alves, M. S., Muñoz Rivera, J. E. and Quintanilla, R. Exponential Decay in Thermoelastic Mixture of Solids. International Journal of Solids and Structures, vol. 46, pp. 1659-1666, 2009.

[25] Muñoz Rivera, J. E., Naso, M. G. and and Quintanilla, R. Decay of Solutions for a Mixture of Thermoelastic One Dimensional Solids. Computers and Mathematics with Applications, vol. 66, pp. 263-277, 2013.

[26] Muñoz Rivera, J. E., Naso, M. G. and and Quintanilla, R. Decay of Solutions for a Mixture of Thermoelastic Solids with Different Temperatures. Computer and Mathematics with Applications, vol. 71, Issue 4, pp. 991-1009, 2016.

[27] Quintanilla, R. Existence and Exponential Decay in the Linear Theory of Viscoelastic Mixtures. European Journal Mechanics A/Solids, vol. 24, pp. 311-324, 2005.

[28] Ieşan, D. and Quintanilla, R. A Theory of Porous Thermoviscoelastic Mixtures, Journal of Thermal Stresses, vol. 30, pp. 693-714, 2007.

[29] Sigillito, V. G. Explicit a priori inequalities with applications to boundary value problems. Pitman Publishing, London, 1977.

[30] Liu, Z. and Zheng, S. Semigroups associated with Dissipative Systems. Chapman and Hall/CRC, 1999. 